OPEN ACCESS

Edited by:

Tanuja Chitnis,

Harvard Medical School,

United States

Reviewed by:

Mattia Rosso,

Harvard Medical School,

United States

Grace Yoonheekim Gombolay,

Emory University, United States

${ }^{*}$ Correspondence:

Qianyi He

fccheqy@zzu.edu.cn

Specialty section:

This article was submitted to

Multiple Sclerosis and

Neuroimmunology,

a section of the journal

Frontiers in Neurology

Received: 25 August 2019 Accepted: 05 November 2019

Published: 21 November 2019

Citation:

Wu K, Wen L, Duan R, Li Y, Yao Y, Jing L, Jia Y, Teng J and $\mathrm{He} Q$ (2019)

Triglyceride Level Is an Independent

Risk Factor in First-Attacked

Neuromyelitis Optica Spectrum

Disorders Patients.

Front. Neurol. 10:1230.

doi: 10.3389/fneur.2019.01230

\section{Triglyceride Level Is an Independent Risk Factor in First-Attacked Neuromyelitis Optica Spectrum Disorders Patients}

\author{
Kaimin Wu, LuLu Wen, Ranran Duan, Yanfei Li, Yaobing Yao, Lijun Jing, Yanjie Jia, \\ Junfang Teng and Qianyi He*
}

Department of Neurology, The First Affiliated Hospital of Zhengzhou University, Zhengzhou, China

Objective: To investigate prospective associations between triglyceride (TG) level and prognosis of first-attacked patients with neuromyelitis optica spectrum disorders (NMOSD).

Methods: This retrospective study included 196 patients newly diagnosed with NMOSD from June 2014 to December 2018. Data of clinical parameters, including age of onset, sex, BMI, blood lipid levels, anti-aquaporin-4 status, serum glucose level, therapy regimens, comorbidities, initial Expanded Disability Status Scale (EDSS), relapses, and outcomes were collected. We used logistic regression models to examine the associations among relevant clinical factors and outcomes, and statistical analyses were performed using the SPSS 23.0 software.

Results: Compared with the high TG group, residual EDSS was relatively lower in the normal TG group (median 1.0 vs. 2.0, $P=0.002$ ). In the univariate analysis, TG level was positively correlated with outcomes (OR 1.75, 95\% Cl 1.18-2.60, $P=0.005$ ) and relapses (OR 1.57, 95\% Cl 1.07-2.31, $P=0.02$ ). Our stratified analysis suggested that patients with normal BMI (OR 4.90, 95\% Cl 2.10-11.44, $P=0.001$ ) were closely correlated with poor recovery owing to increased TG level. In the multivariate analysis, a statistically significant association still existed between TG level and outcomes (OR 3.44, 95\% $\mathrm{Cl} 1.02-11.64 ; P=0.040$ ) after adjusting for various variables.

Conclusions: In first-attacked NMOSD patients, TG level was positively associated with poor recovery. Early monitoring and treatment of elevated TG level in NMOSD patients are important.

Keywords: neuromyelitis optica spectrum disorders, the first-attacked patients, triglyceride level, outcomes, relapse

\section{INTRODUCTION}

Neuromyelitis optica spectrum disorders (NMOSD) is a type of autoimmune demyelinating disease that results in inflammatory lesions in the optic nerves, spinal cord, and other areas of the central nerve system (CNS) (1). Although NMOSD is a rare disorder, it can cause serious disability partly because of high recurrence risk and progressive disability (2). In the last decade, efforts have been 
made to understand the potential pathogenic mechanisms and identify modifiable risks factors to explore effective preventative measures to cure this disease. Approximately $80 \%$ NMO cases are seropositive for aquaporin-4 IgG (3), and myelin oligodendrocyte glycoprotein IgG is also a highly specific diagnostic marker for NMOSD (4). Besides these autoantibodies, many other factors, such as vitamin D level (5), hormone level (6), gastrointestinal infection (7), pregnancy (8), and diet (9), are also involved in the pathological process of NMOSD.

Dyslipidemia, a common comorbidity of cardiovascular diseases (10), has been proposed to be related to the onset and progression of various autoimmune diseases. The values of total cholesterol (TC), low-density lipoprotein (LDL), and triglyceride (TG) during the flare period of systemic lupus erythematosus were all higher than those in the remission stage (11). A largescale observational research found that higher TC level was linked to worsening disability in those suffering from multiple sclerosis (MS) (12). There were also a positive association between TC levels and disability scores in patients with relapsingremitting form of MS (13). Another research suggested that high TG level increases the probability of recurrence of MS (14).

There are limited studies on whether lipid-related variables affect disease progression and prognosis in patients with NMOSD. This study aimed to assess the association between lipid profile (especially TG level) and outcomes in patients newly diagnosed with NMOSD.

\section{MATERIALS AND METHODS}

\section{Patients}

Between June 2014 and December 2018, 846 consecutive patients admitted to the First Affiliated Hospital of Zhengzhou University and newly diagnosed with NMOSD participated in this cohort study. All patients fulfilled the latest diagnostic criteria for NMOSD (1). Inclusion criteria for the NMOSD cohort were as follows: (A) enrolled cases must be confirmed as the firstattacked NMOSD patients by a professional expert of neurology, (B) without hepatic disease, (C) subjects were not prescribed lipid-lowering drugs prior to admission, but those who received statin treatment during the course of this disease were included. (D)patients did not receive immunomodulatory treatment within 6 months prior to admission, and (E) patients who relapsed within 6 months before last follow-up or with incomplete data were excluded. The participants selection process is shown in Figure 1.

\section{Data Collection}

Medical records of all subjects were retrospectively reviewed. Baseline clinical information including age of onset, sex, height, weight, blood lipid levels, anti-aquaporin-4 (AQP4) status, serum glucose level, clinical symptoms, therapy regimens, and comorbidities were collected. Expanded Disability Status Scale (EDSS) at the time of the first attack was carefully evaluated by a professional neurologist and recorded as initial EDSS. Baseline EDSS before the first attack was considered to be normal in all cases.

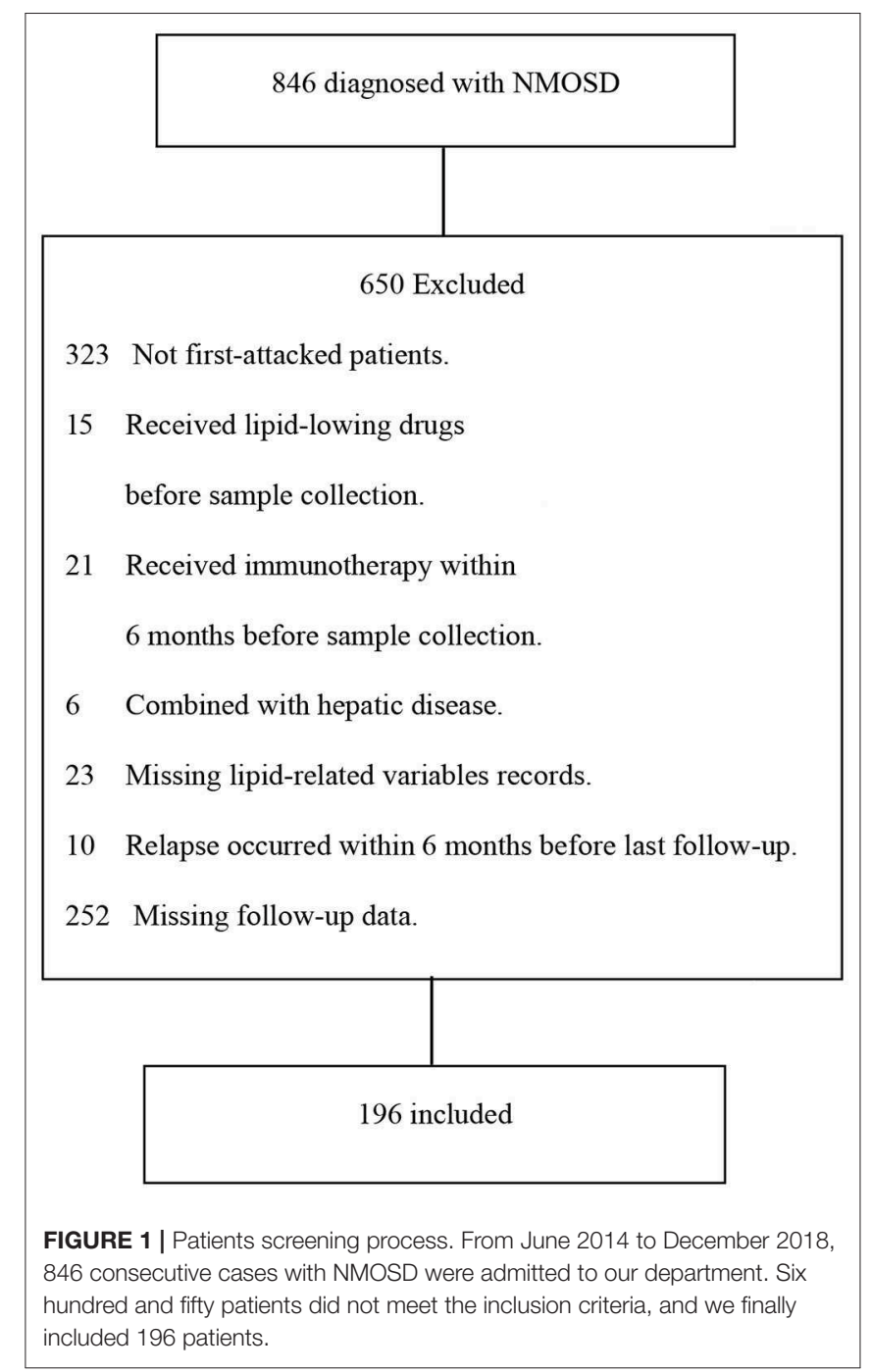

\section{Biomarker Measurement}

The analysis of blood samples was performed at the Biochemistry Laboratory of the First Affiliated Hospital of Zhengzhou University. When more than one fasting lipid test was performed during hospitalization, data from the sampling closest to admission were considered. Fasting TC, LDL, HDL, TG, and glucose levels were evaluated using enzymatic kits (SigmaAldrich). All testing was carried out in accordance with the manufacturer's protocols and the examiners were blinded to diagnoses or clinical symptoms.

\section{Anti-AQP4 Status}

Serum or cerebrospinal fluid samples were used to detect antiAQP4 status at the Neurology Laboratory of the First Affiliated Hospital of Zhengzhou University, using an assay on live cells transfected with AQP4.

\section{Outcomes}

Clinical outcomes in patients at the last follow-up were recorded as residual EDSS. Relapse events and residual EDSS score in 
last follow-up were obtained during clinic visits or via telephone interview. To understand the associations between TG level and clinical outcomes, the residual EDSS variable was rendered into categorical variables. The subjects were divided into two groups according to the residual EDSS scores. We defined subjects with residual EDSS scores above 3 (EDSS > 3) as patients with poor recovery, and the rest were defined as patients with good recovery. Relapses were defined as per the $2001 \mathrm{McD}$ nald criteria as the new onset or recurrent neurological symptoms (lasting for at least $24 \mathrm{~h}$ ) in the absence of fever or infection (15), and the interval time referred to the time to first relapse.

\section{Statistical Analysis}

Data analysis was conducted using the SPSS 23.0 software (International Business Machines Corporation, Chicago, IL, USA). Continuous data were presented as the mean \pm SD if the Kolmogorov-Smirnov test showed that they were normally distributed; otherwise, the data were expressed as median (IQR). Comparisons of means between continuous demographic variables were performed by $t$-test or Kruskal-Wallis test. Categorical variables were indicated in terms of frequency (percentage, \%) and compared using the chi-square or Fisher's exact test. Univariate logistic regression models were used to identify whether TG level or other covariates had an independent effect on outcomes. The covariates are as follows: age of onset, sex, BMI, glucose level, TC, TG, LDL, HDL, AQP4 status, initial EDSS, autoimmune diseases, hypertension, diabetes, therapeutic regimens (including steroids, immunosuppressive therapy, rehabilitation training, and statin treatment), and relapses. Interaction and stratified analysis were employed to examine the association between TG level and the outcomes according to variables mentioned above and continuous data were grouped into dichotomous variables, including age $(\leq 60$ and $>60$ year), BMI $\left(\leq 25\right.$ and $\left.>25 \mathrm{~kg} / \mathrm{m}^{2}\right)(16)$, TC $(\leq 5.0$ and $>5.0 \mathrm{mmol} / \mathrm{L}), \mathrm{HDL}(\leq 1.0$ and $>1.0 \mathrm{mmol} / \mathrm{L}), \mathrm{LDL}(\leq 3.0$ and $>3.0 \mathrm{mmol} / \mathrm{L})(17)$, Glucose $(\leq 6.1$ and $>6.1 \mathrm{mmol} / \mathrm{L})(18)$, and Initial EDSS ( $\leq 3$ and $>3$ ). For multivariate logistic regression, we adjusted multiple confounders to analyze the stability of the associations between TG level and outcomes. Variables, including TC, LDL, and HDL, were considered in the basic model, because lipoproteins are all involved in lipid metabolism. Previous studies demonstrated that age of onset, sex, BMI, statin treatment, initial

TABLE 1 | Characteristics of the patients with first attacked NMOSD according to TG group.

\begin{tabular}{|c|c|c|c|c|}
\hline Clinical characteristics & $\begin{array}{c}\text { Total patients } \\
(n=196)\end{array}$ & $\begin{array}{l}\text { Normal-TG group } \\
\qquad(n=156)\end{array}$ & $\begin{array}{l}\text { High-TG group } \\
\quad(n=40)\end{array}$ & $P$-value \\
\hline Age of onset, years, mean \pm SD & $43.4 \pm 13.6$ & $43.1 \pm 14.8$ & $45.1 \pm 13.9$ & 0.43 \\
\hline Sex, female, $n(\%)$ & $124(63.3)$ & 99 (63.5) & $25(62.5)$ & 0.91 \\
\hline BMI, (Kg/m²), median (IQR) & $22.9(20.4-25.4)$ & $22.8(20.3-25.3)$ & $23.1(20.6-25.4)$ & 0.86 \\
\hline TC, median (IQR), mmol/L & $4.3(3.7-5.0)$ & $4.3(3.6-4.9)$ & $5.0(4.0-5.7)$ & $0.005^{\star}$ \\
\hline $\mathrm{LDL}$, mean $\pm \mathrm{SD}, \mathrm{mmol} / \mathrm{L}$ & $2.7(2.2-3.4)$ & $2.7 \pm 0.6$ & $3.0 \pm 1.2$ & 0.15 \\
\hline Glucose, median (IQR), mmol/L & $4.7(4.2-5.7)$ & $4.9(4.2-5.8)$ & $4.4(4.2-5.8)$ & 0.55 \\
\hline Initial EDSS, median (IQR) & $4.0(3.0-6.0)$ & $4.0(3.0-6.0)$ & $5.0(3.8-6.5)$ & 0.12 \\
\hline \multicolumn{5}{|l|}{ Anti-AQP4 status, $n(\%)$} \\
\hline Negative & $29(31.2)$ & $22(29.7)$ & $7(36.8)$ & 0.53 \\
\hline Autoimmune diseases, $\mathrm{n}(\%)$ & $10(5.1)$ & $8(5.1)$ & $2(5.0)$ & 0.97 \\
\hline Corticosteroid treatment, n (\%) & $180(91.8)$ & $143(91.7)$ & $37(92.5)$ & 0.86 \\
\hline Immunosuppressant treatment, $n(\%)$ & $56(28.6)$ & $42(26.9)$ & $14(35.0)$ & 0.31 \\
\hline Rehabilitation training, $n(\%)$ & 39 (19.9) & $30(19.2)$ & $9(22.5)$ & 0.64 \\
\hline Statin treatment, $n(\%)$ & $21(13.5)$ & $15(9.9)$ & $6(15 \%)$ & 0.48 \\
\hline Residual EDSS, median (IQR) & $1.5(0.5-3.5)$ & $1.0(0-3.0)$ & $2.0(1.8-4.0)$ & $0.002^{*}$ \\
\hline Outcomes, good recovery, $n$ (\%) & $142(72.4)$ & $122(78.2)$ & $20(50.0)$ & $0.006^{\star}$ \\
\hline Relapses, $n$ (\%) & $72(36.7)$ & $53(40.0)$ & $19(47.5)$ & 0.11 \\
\hline Interval time, month, median (IQR) & $9.2(6.3-15.2)$ & $10.0(6.3-15.0)$ & $7.0(6.3-12.0)$ & 0.08 \\
\hline Follow-up time, month, median (IQR) & $20.5(13.4-37.1)$ & $21.2(15.0-40.4)$ & $20.3(11.6-36.4)$ & 0.54 \\
\hline
\end{tabular}

Continuous variables were presented as mean $\pm S D$ or median (IQR $=25$ th-75th percentile), and categorical variables were described as percentages.

Normal-TG Group, patients whose triglyceride levels were below or equal to $1.7 \mathrm{mmol} / \mathrm{L}$; High-TG Group, patients whose triglyceride levels were higher than $1.7 \mathrm{mmol} / \mathrm{L}$.

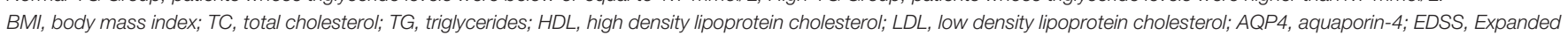
Disability Status Scale.

${ }^{*} p<0.05$. 
EDSS, and relapse were significantly associated with outcomes in NMOSD (19-21); hence, Adjust-I model further adjusted for these factors. We finally examined the associations between TG level and outcomes by furtherly adjusting for glucose levels, treatment methods, and complications in Adjusted II model. The significance level was established at $p<0.05$.

\section{RESULTS}

\section{Demographic and Clinical Characteristics}

In this cohort study, a total of 196 cases with newly diagnosed NMOSD were enrolled and the detailed screening process is shown in Figure 1. The demographic and clinical characteristics of all patients are presented in Table 1. To better understand the effect of TG level on clinical prognosis, we classified patients into two groups according to serum TG level: normal TG group (TG $\leq 1.7 \mathrm{mmol} / \mathrm{L}$ ) and high $\mathrm{TG}$ group ( $\mathrm{TG}>1.7$ $\mathrm{mmol} / \mathrm{L}$ ). Compared with the high TG group, levels of TC were dramatically lower (median 4.3 vs. 5.0, $P=0.005$ ), and those of TG also remarkedly decreased (median 1.0 vs. $2.3, P<$ 0.001 ), while those of HDL significantly increased in the normal TG group (median 1.3 vs. $1.1, P=0.003$ ). Other parameters, including age at onset, ratio of female patients, BMI, LDL and glucose levels remained unchanged. Clinical manifestations of all 196 patients were carefully evaluated at first attack and recorded as initial EDSS, but no significant differences were observed between the two groups. Only half the patients were tested for anti-AQP4 status in cerebrospinal fluid or serum, and the positive results in both groups were more than $60 \%$. There were also no significant differences in the number of patients who suffered from hypertension, diabetes or autoimmune diseases (including systemic lupus erythematosus, sicca syndrome, IgA nephropathy, and thyroiditis). Considering clinical symptoms and financial situations, patients received different treatments, such as corticosteroids, immunosuppressants (azathioprine, rituximab, and mycophenolate mofetil), rehabilitation training, and statin treatment, but no significant differences emerged between the two groups. At the last follow-up, the residual EDSS scores were significantly lower in the normal TG group than in the high TG group (median 1.0 vs. $2.0, P=0.002$ ), and the proportion of patients who gained a good recovery was higher in the normal TG group than in the high TG group (78.2 vs. $50.0 \%$, $P=0.006)$. Approximately $36 \%$ of patients relapsed during the follow-up period, and the interval time was left skewed with a median time 9.2 months (Figure 2), but relapses rate and the interval time between two groups during follow-up were not markedly different.

\section{Effects of Clinical Parameters on the Prognosis of NMOSD Patients}

To explore potential factors that may affect the prognosis of patients, univariate logistic analysis was performed. As shown in Table 2, TG level was positively associated with an increased risk of poor recovery (OR 1.75, 95\% CI 1.18-2.60, $P=0.005)$. Age of onset (OR 1.03, 95\% CI 1.00-1.12, $P=0.024$ ), AQP4 status (OR 1.44, 95\% CI 1.02-2.04, $P=0.037$ ), diabetes (OR 3.97, 95\% CI

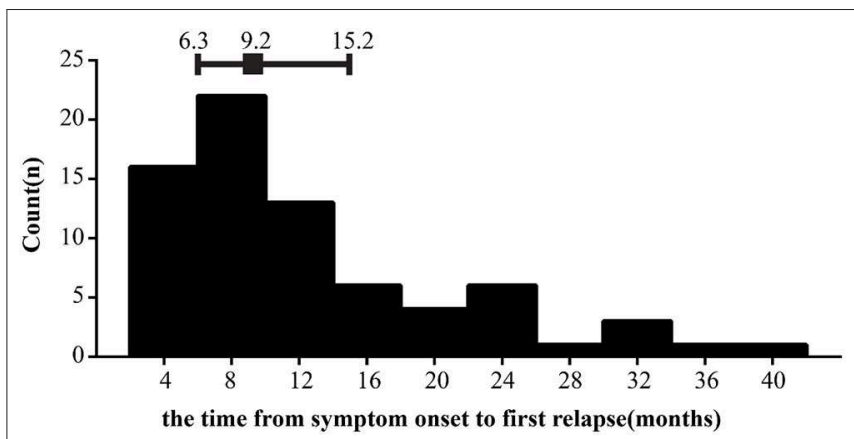

FIGURE 2 | Distributions of the interval time from symptom onset to first relapse. Error bars show median surrounded by 25th and 75 th percentiles.

TABLE 2 | Univariate logistic regression analysis of factors associated with the outcomes of NMOSD patients.

\begin{tabular}{|c|c|c|}
\hline \multirow[t]{2}{*}{ Variable } & \multicolumn{2}{|c|}{ Outcomes } \\
\hline & OR (95\% Cl) & $P$-value \\
\hline Age of onset & $1.03(1.00-1.12)$ & $0.024^{*}$ \\
\hline Sex & $1.52(0.84-2.74)$ & 0.17 \\
\hline BMl & $1.01(0.92-1.12)$ & 0.66 \\
\hline TC & $1.09(0.82-1.45)$ & 0.56 \\
\hline TG & $1.75(1.18-2.60)$ & $0.005^{\star}$ \\
\hline $\mathrm{HDL}$ & $0.63(0.26-1.53)$ & 0.31 \\
\hline LDL & $1.20(0.85-1.70)$ & 0.38 \\
\hline Glucose level & $1.13(0.96-1.33)$ & 0.15 \\
\hline${ }^{\mathrm{a}} \mathrm{AQP} 4$ status & $1.44(1.02-2.04)$ & $0.037^{*}$ \\
\hline Hypertension & $1.60(0.55-4.65)$ & 0.38 \\
\hline Diabetes & $3.97(1.20-13.09)$ & $0.049^{*}$ \\
\hline Autoimmune disease & $0.63(0.13-3.05)$ & 0.56 \\
\hline Corticosteroid treatment & $0.68(0.36-2.67)$ & 0.43 \\
\hline Immunosuppressant treatment & $0.91(0.45-1.82)$ & 0.7 \\
\hline Rehabilitation training & $1.44(0.67-3.06)$ & 0.84 \\
\hline Statin treatment & $2.64(1.05-6.06)$ & $0.04^{*}$ \\
\hline Initial EDSS & 1.59 (1.29-1.90) & $0.001^{*}$ \\
\hline Relapse & $2.21(1.17-4.18)$ & $0.023^{*}$ \\
\hline bTG & $1.57(1.07-2.31)$ & $0.02^{*}$ \\
\hline
\end{tabular}

BMI, body mass index; TC, total cholesterol TG, triglycerides; HDL, high density lipoprotein cholesterol; LDL, low density lipoprotein cholesterol; AQP4, aquaporin-4; EDSS, Expanded Disability Status Scale; OR, Odds ratio; Cl, confidence interval.

${ }^{a}$ Not-tested patients $(n=93)$ were excluded.

${ }^{b}$ The associations between TG level and relapses were analyzed. ${ }^{*} p<0.05$.

$1.20-13.09, P=0.049)$, statin treatment (OR 2.64, 95\% CI 1.05$6.06, P=0.04$ ) initial EDSS (OR 1.59, 95\% CI 1.29-1.90, $P=$ 0.001 ), and relapses (OR 2.21, 95\% CI, 1.17-4.18, $P=0.023$ ) were significantly associated with outcomes in patients. Sex (OR 1.52, 95\% CI 0.84-2.74, $P=0.17$ ) and glucose level (OR 1.13, 95\% CI $0.96-1.33, P=0.15$ ) had a moderate impact, but TC, HDL, LDL, BMI, hypertension, autoimmune diseases, and treatment methods were not associated with the outcomes. In addition, we observed a statistically significant correlation between TG level and relapse (OR 1.57,95\% CI 1.07-2.31, $P=0.02$ ). 


\section{Associations Between TG Level and Prognosis of NMOSD Patients According to Clinical Parameters}

In order to further analyze the impact of various parameters on the association between TG level and prognosis, we subsequently divided the variables into different subgroups and conducted subgroup and interaction analysis. The results in Table 3 indicated that TG level had a positive association with poor outcomes according to all variables. Notably, we found significant heterogeneity between the subgroups analyzed according to BMI and corticosteroid treatment. As TG level increased, the risk of disability was obviously higher in patients with normal BMI (OR 4.90, 95\% CI 2.10-11.44; $P=0.001)$ than those with high BMI (OR 0.93, 95\% CI $0.20-3.40, P=0.79$ ). Compared to subjects without receiving corticosteroid treatment (OR 19.26, 95\% CI $0.75-493.74, P=0.07)$, those who treated with corticosteroid (OR 1.59, 95\% CI 1.06-2.37, $P=0.024$ ) had significantly lower chances of poor recovery because of elevated TG level.

\section{TG Level Is a Predictor of the Outcomes of the First-Attacked NMOSD Patients}

In the basic multivariate logistic regression modeling (Table 4), a higher TG level at study entry demonstrated a lower probability to regain good outcomes (OR 6.7, 2.12-21.18; $P=0.001$ ). This association remained robust after adjusting for age of onset, sex, BMI, statin treatment initial EDSS, and relapses (OR 3.33;1.9911.15; $P=0.02$ ) in Adjusted I model. Subsequently, we further adjusted for BMI, glucose levels, complications and treatment methods in Adjusted-II model, but there still existed a statistically significant association between TG level and outcomes (OR 3.44; $1.02-11.64 ; P=0.04)$.

\section{DISCUSSION}

NMOSD is a chronic autoimmune demyelinating disorder that generally leads to severe and irreversible disabilities including blindness and paralysis (22), but reliable prognostic indicators for this deleterious disease are still lacking. In this cohort study, we examined the possible associations of lipid parameters with disease progression through a large NMOSD dataset. The results indicated that TG level was positively correlated with clinical outcomes, and therefore, we speculated that TG level might have a prognosticative value for patients newly diagnosed with NMOSD. But levels of HDL, LDL, and TC did not relate to outcomes. To our best knowledge, this is the first report to demonstrate that high TG level may worsen disability in NMOSD.

As a key storage molecule of metabolic energy and fatty acids, TG is involved in many biological processes through mediation of lipogenesis or lipolysis, which are essential for organs to maintain normal function $(23,24)$. Emerging evidence indicate that TG metabolism pathways closely interacted with activated immune system (25-27). One possible mechanism was that excessive accumulation of TG caused metabolic disturbance and lipid deposition in lymphoid tissues, and those pathological conditions triggered immune activation (25). Additionally, TG was a leukocyte activator that stimulated inflammation by increasing the expression of antigen markers on surface of leukocytes (28). A recent clinical trial based on general US population suggested that elevated TG implied increased leukocyte profiles and lipidlowering intervention might exert beneficial anti-inflammatory and immunomodulatory effects (29). In turn, inflammatory cytokines could also alter serum TG level (30). Previous studies showed that both tumor necrosis factor and interleukin-l could induce a remarkable increase in serum TG level during infection and inflammation process (31).

Among autoimmune demyelination diseases of the CNS, patients with MS and NMO had higher serum TG levels than healthy controls (32). Elevated TG levels was able to increase IL-6 concentrations in cerebrospinal fluid (16) and augment the risk of subsequent relapse in participants after a first demyelinating event (33), while lower serum TG level and BMI could ameliorate central inflammation and reduce the accumulation of disability in relapsing-remitting MS patients (21). During the pathological process of MS, higher lipoproteins can aggravate inflammation at vascular endothelium, leading to immune cells across the activated endothelium of blood brain barrier (14). There is no data on how TG plays a role in the pathogenesis and development of NMOSD. NMOSD was considered as a severe variant of MS, and various types of immune cells [including regulatory $\mathrm{T}$ cells (34), neutrophils (35), and B lymphocytes (36)] and cytokines [including IL-6 (37), IFN (38), and IL-10 (34)] are involved in disease onset and progression. It has been proposed that CNS inflammation mediated by cytokines may exacerbate the clinical status of NMO (39). Therefore, we speculated that TG might indirectly reflect the inflammatory status by interacting with immune responses in NMOSD patients, but this assumption needs further research.

In our study, patients who were newly diagnosed with NMOSD were eligible, allowing us to eliminate the effects of previous treatment on outcome events. All subjects were divided into two groups according to TG level at admission. We found that patients with high TG level $(\geq 1.7 \mathrm{mmol} / \mathrm{L})$ were more likely to have increased TC and LDL values, while the HDL levels significantly decreased (Table 1), which indicated that dyslipidemia also exists in patients with NMOSD. This finding was in line with a case-control study regarding lipoproteins in NMO showing that NMO patients have elevated TC, TG, and LDL levels compared with those in healthy controls (32).

The average age of onset was 43.4 years in our study, which is comparable another previous study regarding epidemiology of NMOSD in Sweden (19). A recent research suggested that age of onset may be a prognostic factor for NMOSD, in which lower aged patients with NMOSD had a higher chance of good recovery after receiving immunoadsorption treatment (40). In line with this research, our univariate analysis also found that age of onset was positively associated with outcomes in firstattacked NMOSD patients. AQP4-IgG was a specific biomarker for the diagnosis of $\mathrm{NMO}$, which can cause demyelination and neurologic deficit by binding to astrocytes and leading to astrocyte injury (41). Positive AQP4 status was closely related to rapid progress, severe tissue damage, and abundant infiltration of leukocyte in some NMO patients (42). Consistent with results 
TABLE 3 | Association between TG and the outcomes according to baseline characteristics.

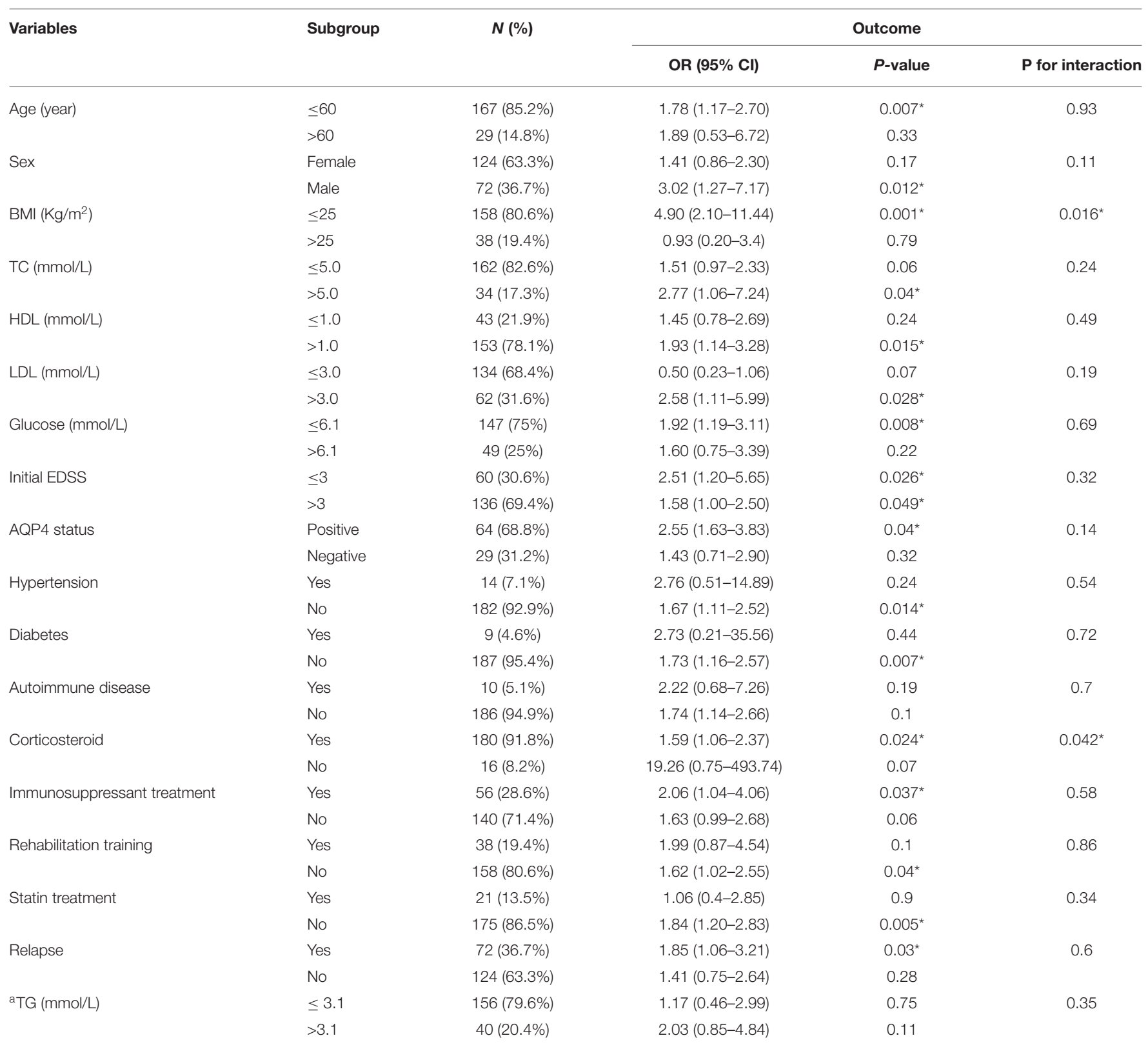

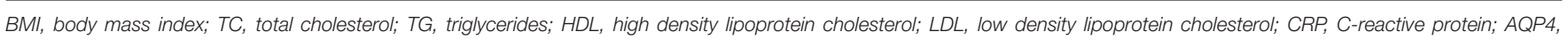
aquaporin-4; EDSS, Expanded Disability Status Scale; OR, Odds ratio; Cl, confidence interval.

${ }^{a}$ The associations between TG level and relapses according to TG categories.

${ }^{*} p<0.05$.

of previous studies, our results suggested that AQP4 status had positive correlations with outcomes of NMOSD cases. No significant association between BMI and outcomes was observed in our study, while a cohort study showed that BMI was a predictive factor of EDSS worsening for patients with refractory NMO who were treated with rituximab, but only 21 subjects were included in this research (20). Statin has been reported to have anti-inflammatory and immunomodulatory effects in an experimental mouse model of NMO by increasing CD55 expression (43), and our data also support that statin treatment on the disease courses has a significant correlation to outcomes. Since long-term disability depends on initial symptoms and the accumulation of disability after several attacks $(21,44,45)$, we also examined the correlations among initial EDSS, relapse, and outcomes. Accordingly, our findings proved that the initial EDSS and relapses can affect the prognosis of patients, suggesting the importance of early prevention and effective treatment. Previous evidence also suggested that TG level showed significant 
TABLE 4 | Adjusted logistic regression models of lipid parameters associated with the outcomes of NMOSD patients.

\begin{tabular}{|c|c|c|c|c|c|c|}
\hline \multirow[t]{2}{*}{ Variable } & \multicolumn{2}{|c|}{ Basic modela } & \multicolumn{2}{|c|}{ Adjust-I model ${ }^{b}$} & \multicolumn{2}{|c|}{ Adjust-II model ${ }^{c}$} \\
\hline & OR $(95 \% \mathrm{Cl})$ & $P$-value & OR $(95 \% \mathrm{Cl})$ & $P$-value & OR (95\% Cl) & $P$-value \\
\hline TG & $6.70(2.12-21.18)$ & $0.001^{*}$ & 3.33 (1.99-11.15) & $0.02^{\star}$ & $3.44(1.02-11.64)$ & $0.04^{*}$ \\
\hline TC & $1.98(0.30-13.08)$ & 0.48 & $7.38(0.72-75.34)$ & 0.09 & $7.57(0.70-81.24)$ & 0.1 \\
\hline HDL & $0.75(0.10-5.74)$ & 0.78 & 0.29 (0.03-2.90) & 0.29 & 0.39 (0.04-4.26) & 0.44 \\
\hline LDL & $0.24(0.04-1.65)$ & 0.24 & $0.06(0.05-1.52)$ & 0.21 & $0.07(0.05-1.21)$ & 0.14 \\
\hline Statin treatment & & & $3.34(0.58-19.10)$ & 0.18 & $2.81(0.47-16.89)$ & 0.26 \\
\hline Age of onset & & & $1.02(0.07-1.06)$ & 0.53 & $1.01(0.96-1.05)$ & 0.82 \\
\hline Sex & & & $1.52(0.51-4.55)$ & 0.95 & $1.80(0.54-6.05)$ & 0.34 \\
\hline BMl & & & $0.95(0.80-1.13)$ & 0.57 & $0.96(0.79-1.15)$ & 0.63 \\
\hline Initial EDSS & & & $1.74(1.26-2.42)$ & $0.001^{*}$ & $1.90(1.31-2.77)$ & $0.001^{\star}$ \\
\hline Relapse & & & $3.81(1.15-12.60)$ & $0.03^{*}$ & $4.51(1.24-16.43)$ & $0.02^{*}$ \\
\hline Glucose level & & & & & $1.05(0.76-1.45)$ & 0.77 \\
\hline Hypertension & & & & & $0.47(0.07-3.22)$ & 0.47 \\
\hline Diabetes & & & & & $5.81(0.68-49.88)$ & 0.11 \\
\hline Autoimmune disease & & & & & $2.62(0.20-34.82)$ & 0.47 \\
\hline Corticosteroid treatment & & & & & $1.24(0.11-13.82)$ & 0.86 \\
\hline Immunosuppressant treatment & & & & & $1.36(0.37-4.97)$ & 0.64 \\
\hline Rehabilitation training & & & & & $1.83(0.45-7.38)$ & 0.39 \\
\hline
\end{tabular}

a Basic model: Adjusted for TC, LDL, HDL.

${ }^{b}$ Adjust-I model: Further adjusted for statin treatment, BMI, relapse, age, sex, and Initial EDSS.

${ }^{C}$ Adjust-II model: Further adjusted for glucose level, hypertension, diabetes, autoimmune disease, corticosteroid, Rehabilitation, and immunosuppressant.

BMI, body mass index; TC, total cholesterol; TG, triglycerides; HDL, high density lipoprotein cholesterol; LDL, low density lipoprotein cholesterol; CRP, C-reactive protein; EDSS, Expanded Disability Status Scale; OR, Odds ratio; Cl, confidence interval.

correlation with relapses in MS (14); therefore, we examined whether this association existed in patients with NMOSD. Consistent with results of the previous research, our results indicated that high TG level increased the risk of relapse. In our cohort, the risk for first relapse was highest in the first year after disease onset (median 9.2 months) (19), while this figure was slightly larger with a median 1.42 year in a 26 -year nationwide population-based study, which we attributed to the relatively short follow-up time in our study.

A matched case-control study of NMO suggested that a lower BMI was associated with a higher mortality in women (46), and one of the potential mechanisms hypothesized by this research was that a lower BMI could argument the susceptibility to autoimmunity because of the deregulated level of adipokines or sex hormones $(47,48)$. Interestingly, our subgroup and interaction analysis showed that patients with normal BMI displayed a dramatically higher risk of adverse outcomes than those with higher BMI as TG level elevated, but the mechanisms about how BMI interacts with the TG biological pathway and worsen disease progression in NMOSD still unclear. Since only 38 patients were included in the higher BMI group, the results may be biased and need to be further verified in a larger sample size study.

Some limitations of our study need to be addressed further. Because the total number of subjects in our cohort study was small and patients were from a single center with a relatively short follow-up duration, the results need to be further validated in larger, multicenter, and long follow-up duration studies. Previous studies suggested that low dairy consumption and lack of physical activity were potential risk factors for $\operatorname{NMOSD}(9,49)$, but we failed to include these two factors in our retrospective study due to the majority of patients providing inaccurate data on their daily diet and exercise habits, which may bias our results. In addition, it is essential to explore the role of TG pathway in the progression of NMOSD. In conclusion, our study suggests that a significant association exists between TG level and outcomes in NMOSD, and early monitoring and treatment of adverse lipid profiles in NMOSD may be necessary.

\section{DATA AVAILABILITY STATEMENT}

The datasets generated for this study are available on request to the corresponding author.

\section{ETHICS STATEMENT}

The studies involving human participants were reviewed and approved by the Ethics Committee of Zhengzhou University. The patients/participants provided their written informed consent to participate in this study.

\section{AUTHOR CONTRIBUTIONS}

KW and QH were responsible for conceiving and designing the study and drafted the manuscript. LW, RD, and YL 
conducted the regular follow-up of all cases. YJ and JT undertook the task of assessing the initial EDSS and residual EDSS scores of all patients. YY, QH, KW, and LJ conducted the data analysis. All authors were involved in the process of data collection, reviewed, and approved the final submitted manuscript.

\section{REFERENCES}

1. Wingerchuk DM, Banwell B, Bennett JL, Cabre P, Carroll W, Chitnis $\mathrm{T}$, et al. International consensus diagnostic criteria for neuromyelitis optica spectrum disorders. Neurology. (2015) 85:177-89. doi: 10.1212/WNL.0000000000001729

2. Stellmann Jp, Krumbholz M, Friede T, Gahlen A, Borisow N, Fischer K, et al. Immunotherapies in neuromyelitis optica spectrum disorder: efficacy and predictors of response. J Neurol Neurosurg Psychiatry. (2017) 88:639-47. doi: 10.1136/jnnp-2017-315603

3. Bruscolini A, Sacchetti M, La Cava M, Gharbiya M, Ralli M, Lambiase A, et al. Diagnosis and management of neuromyelitis optica spectrum disorders - an update. Autoimmun Rev. (2018) 17:195-200. doi: 10.1016/j.autrev.2018.01.001

4. Petzold A, Woodhall M, Khaleeli Z, Tobin WO, Pittock SJ, Weinshenker BG, et al. Aquaporin-4 and myelin oligodendrocyte glycoprotein antibodies in immune-mediated optic neuritis at long-term follow-up. J Neurol Neurosurg Psychiatry. (2019) 90:1021-6. doi: 10.1136/jnnp-2019-320493

5. Koduah P, Paul F, Dörr Jm. Vitamin D in the prevention, prediction and treatment of neurodegenerative and neuroinflammatory diseases. EPMA J. (2017) 8:313-25. doi: 10.1007/s13167-017-0120-8

6. Pereira WL, Reiche EM, Kallaur AP, Kaimen-Maciel Dr. Epidemiological, clinical, and immunological characteristics of neuromyelitis optica: a review. J Neurol Sci. (2015) 355:7-17. doi: 10.1016/j.jns.2015.05.034

7. Yoshimura S, Isobe N, Matsushita T, Yonekawa T, Masaki K, Sato S, et al. Distinct genetic and infectious profiles in Japanese neuromyelitis optica patients according to anti-aquaporin 4 antibody status. J Neurol Neurosurg Psychiatry. (2013) 84:29-34. doi: 10.1136/jnnp-2012-302925

8. Klawiter EC, Bove R, Elsone L, Alvarez E, Borisow N, Cortez M, et al. High risk of postpartum relapses in neuromyelitis optica spectrum disorder. Neurology. (2017) 89:2238-44. doi: 10.1212/WNL.0000000000004681

9. Rezaeimanesh N, Razeghi Jahromi S, Ghorbani Z, Beladi Moghadam N, Hekmatdoost A, Naser Moghadasi A, et al. The association between dietary sugar intake and neuromyelitis optica spectrum disorder: a case-control study. Mult Scler Relat Disord. (2019) 31:112-7. doi: 10.1016/j.msard.2019.03.028

10. Ye X, Kong W, Zafar MI, Chen LL. Serum triglycerides as a risk factor for cardiovascular diseases in type 2 diabetes mellitus: a systematic review and meta-analysis of prospective studies. Cardiovasc Diabetol. (2019) 18:48. doi: 10.1186/s12933-019-0851-z

11. Urquizu-Padilla M, Balada E, Chacon P, Pérez EH, Vilardell-Tarrés M, OrdiRos J. Changes in lipid profile between flare and remission of patients with systemic lupus erythematosus: a prospective study. J Rheumatol. (2009) 36:1639-45. doi: 10.3899/jrheum.081097

12. Zhornitsky S, Mckay KA, Metz LM, Teunissen CE, Rangachari M. Cholesterol and markers of cholesterol turnover in multiple sclerosis: relationship with disease outcomes. Mult Scler Relat Disord. (2016) 5:53-65. doi: 10.1016/j.msard.2015.10.005

13. Durfinová $M$, Procházková L, Petrleničová $\mathrm{D}$, Bystrická $\mathrm{Z}$, Orešanská $\mathrm{K}$, Kuračka L, et al. Cholesterol level correlate with disability score in patients with relapsing-remitting form of multiple sclerosis. Neurosci Lett. (2018) 687:304-7. doi: 10.1016/j.neulet.2018.10.030

14. Palavra F, Marado D, Mascarenhas-Melo F, Sereno J, Teixeira-Lemos E, Nunes CC, et al. New markers of early cardiovascular risk in multiple sclerosis patients: oxidized-LDL correlates with clinical staging. Dis Mark. (2013) 34:341-8. doi: 10.1155/2013/567162

15. Mcdonald WI, Compston A, Edan G, Goodkin D, Hartung HP, Lublin FD, et al. Recommended diagnostic criteria for multiple sclerosis: guidelines from the International Panel on the diagnosis of multiple sclerosis. Ann Neurol. (2001) 50:121-7. doi: 10.1002/ana.1032

16. Stampanoni Bassi M, Iezzi E, Buttari F, Gilio L, Simonelli I, Carbone F, et al. Obesity worsens central inflammation and disability in multiple sclerosis. Mult Scler. (2019). doi: 10.1177/1352458519853473. [Epub ahead of print].

17. Kutkiene S, Petrulioniene Z, Laucevicius A, Cerkauskiene R, Staigyte J, Saulyte A, et al. Lipid profile evaluation and severe hypercholesterolaemia screening in the middle-aged population according to nationwide primary prevention programme in Lithuania. Atherosclerosis. (2018) 277:267-72. doi: 10.1016/j.atherosclerosis.2018.06.008

18. Qiu S, Cai X, Yin H, Zügel M, Sun Z, Steinacker JM, et al. Association between circulating irisin and insulin resistance in non-diabetic adults: a meta-analysis. Metab Clin Exp. (2016) 65:825-34. doi: 10.1016/j.metabol.2016.02.006

19. Jonsson DI, Sveinsson O, Hakim R, Brundin L. Epidemiology of NMOSD in Sweden from 1987 to 2013: a nationwide population-based study. Neurology. (2019) 93:e181-e9. doi: 10.1212/WNL.0000000000007746

20. Collongues N, Brassat D, Maillart E, Labauge P, Ouallet JC, Carra-Dalliere C, et al. Efficacy of rituximab in refractory neuromyelitis optica. Mult Scler. (2016) 22:955-9. doi: 10.1177/1352458515602337

21. Tettey P, Simpson S, Taylor B, Blizzard L, Ponsonby AL, Dwyer $\mathrm{T}$, et al. An adverse lipid profile is associated with disability and progression in disability, in people with MS. Mult Scler. (2014) 20:1737-44. doi: $10.1177 / 1352458514533162$

22. Weinshenker BG, Wingerchuk DM. Neuromyelitis spectrum disorders. Mayo Clin Proc. (2017) 92:663-79. doi: 10.1016/j.mayocp.2016.12.014

23. Sparks JD, Sparks CE, Adeli K. Selective hepatic insulin resistance, VLDL overproduction, and hypertriglyceridemia. Arterioscler Thromb Vasc Biol. (2012) 32:2104-12. doi: 10.1161/ATVBAHA.111.241463

24. Reiner Ž. Hypertriglyceridaemia and risk of coronary artery disease. Nat Rev Cardiol. (2017) 14:401-11. doi: 10.1038/nrcardio.2017.31

25. Andersen CJ, Murphy KE, Fernandez ML. Impact of obesity and metabolic syndrome on immunity. Adv Nutr. (2016) 7:66-75. doi: 10.3945/an.115.010207

26. Van De Woestijne AP, Monajemi H, Kalkhoven E, Visseren Fl. Adipose tissue dysfunction and hypertriglyceridemia: mechanisms and management. Obes Rev. (2011) 12:829-40. doi: 10.1111/j.1467-789X.2011.00900.x

27. Calder PC. The relationship between the fatty acid composition of immune cells and their function. Prostaglandins Leukot Essent Fatty Acids. (2008) 79:101-8. doi: 10.1016/j.plefa.2008.09.016

28. Alipour A, Van Oostrom AJ, Izraeljan A, Verseyden C, Collins JM, Frayn $\mathrm{KN}$, et al. Leukocyte activation by triglyceride-rich lipoproteins. Arterioscler Thromb Vasc Biol. (2008) 28:792-7. doi: 10.1161/ATVBAHA.107.159749

29. Andersen CJ, Vance TM. Gender dictates the relationship between serum lipids and leukocyte counts in the national health and nutrition examination survey 1999-2004. J Clin Med. (2019) 8:E365. doi: 10.3390/jcm8030365

30. Nonogaki K, Fuller GM, Fuentes NL, Moser AH, Staprans I, Grunfeld C, et al. Interleukin-6 stimulates hepatic triglyceride secretion in rats. Endocrinology. (1995) 136:2143-9. doi: 10.1210/endo.136.5.7720663

31. Feingold KR, Soued M, Adi S, Staprans I, Neese R, Shigenaga J, et al. Effect of interleukin-1 on lipid metabolism in the rat. Similarities to and differences from tumor necrosis factor. Arterioscler Thromb J Vasc Biol. (1991) 11:495-500. doi: 10.1161/01.ATV.11.3.495

32. Li Y, Wang H, Hu X, Peng F, Yang Y. Serum lipoprotein levels in patients with neuromyelitis optica elevated but had little correlation with clinical presentations. Clin Neurol Neurosurg. (2010) 112:478-81. doi: 10.1016/j.clineuro.2010.03.017

33. Tettey P, Simpson S, Taylor B, Ponsonby AL, Lucas RM, Dwyer T, et al. An adverse lipid profile and increased levels of adiposity significantly predict clinical course after a first demyelinating event. J Neurol Neurosurg Psychiatry. (2017) 88:395-401. doi: 10.1136/jnnp-2016-315037

34. Cho EB, Cho HJ, Seok JM, Min JH, Kang ES, Kim BJ. The IL-10producing regulatory $\mathrm{B}$ cells (B10 cells) and regulatory $\mathrm{T}$ cell subsets in neuromyelitis optica spectrum disorder. Neurol Sci. (2018) 39:543-9. doi: 10.1007/s10072-018-3248-y 
35. Casserly CS, Nantes JC, Whittaker Hawkins RF, Vallières L. Neutrophil perversion in demyelinating autoimmune diseases: mechanisms to medicine. Autoimmun Rev. (2017) 16:294-307. doi: 10.1016/j.autrev.2017.01.013

36. Bennett JL, O'connor KC, Bar-Or A, Zamvil SS, Hemmer B, Tedder TF, et al. B lymphocytes in neuromyelitis optica. Neurol Neuroimmunol Neuroinflamm. (2015) 2:e104. doi: 10.1212/NXI.0000000000000104

37. Chihara N, Aranami T, Sato W, Miyazaki Y, Miyake S, Okamoto T, et al. Interleukin 6 signaling promotes anti-aquaporin 4 autoantibody production from plasmablasts in neuromyelitis optica. Proc Natl Acad Sci USA. (2011) 108:3701-6. doi: 10.1073/pnas.1017385108

38. Axtell RC, Raman C, Steinman L. Interferon- $\beta$ exacerbates Th17mediated inflammatory disease. Trends Immunol. (2011) 32:272-7. doi: 10.1016/j.it.2011.03.008

39. Uzawa A, Mori M, Masahiro M, Kuwabara S. Cytokines and chemokines in neuromyelitis optica: pathogenetic and therapeutic implications. Brain Pathol. (2014) 24:67-73. doi: 10.1111/bpa.12097

40. Lipphardt M, Mühlhausen J, Kitze B, Heigl F, Mauch E, Helms HJ, et al. Immunoadsorption or plasma exchange in steroid-refractory multiple sclerosis and neuromyelitis optica. J Clin Apheresis. (2019) 34:381-91. doi: $10.1002 /$ jca. 21686

41. Duan T, Smith AJ, Verkman AS. Complement-independent bystander injury in AQP4-IgG seropositive neuromyelitis optica produced by antibodydependent cellular cytotoxicity. Acta Neuropathol Commun. (2019) 7:112. doi: 10.1186/s40478-019-0766-7

42. Misu T, Höftberger R, Fujihara K, Wimmer I, Takai Y, Nishiyama S, et al. Presence of six different lesion types suggests diverse mechanisms of tissue injury in neuromyelitis optica. Acta Neuropathol. (2013) 125:815-27. doi: 10.1007/s00401-013-1116-7

43. Tradtrantip L, Duan T, Yeaman MR, Verkman As. CD55 upregulation in astrocytes by statins as potential therapy for AQP4-IgG seropositive neuromyelitis optica. J Neuroinflamm. (2019) 16:57. doi: 10.1186/s12974-019-1448-x
44. Palace J, Lin DY, Zeng D, Majed M, Elsone L, Hamid S, et al. Outcome prediction models in AQP4-IgG positive neuromyelitis optica spectrum disorders. Brain J Neurol. (2019) 142:1310-23. doi: 10.1093/brain/ awz054

45. Kleiter I, Gahlen A, Borisow N, Fischer K, Wernecke KD, Wegner B, et al. Neuromyelitis optica: evaluation of 871 attacks and 1,153 treatment courses. Ann Neurol. (2016) 79:206-16. doi: 10.1002/ana.24554

46. Le TM, Szilasi T, Volford B, Szekeres A, Fülöp F, Szakonyi Z. Stereoselective synthesis and investigation of isopulegol-based chiral ligands. Int J Mol Sci. (2019) 20:E4050. doi: 10.3390/ijms20164050

47. Ouchi N, Parker JL, Lugus JJ, Walsh K. Adipokines in inflammation and metabolic disease. Nat Rev Immunol. (2011) 11:85-97. doi: 10.1038/n ri2921

48. Ansar Ahmed S, Penhale WJ, Talal N. Sex hormones, immune responses, and autoimmune diseases. Mechanisms of sex hormone action. Am J Pathol. (1985) 121:531-51.

49. Eskandarieh S, Nedjat S, Abdollahpour I, Azimi AR, Moghadasi AN, Asgari $\mathrm{N}$, et al. Environmental risk factors in neuromyelitis optica spectrum disorder: a case-control study. Acta Neurol Belgica. (2018) 118:277-87. doi: 10.1007/s13760-018-0900-5

Conflict of Interest: The authors declare that the research was conducted in the absence of any commercial or financial relationships that could be construed as a potential conflict of interest.

Copyright (c) 2019 Wu, Wen, Duan, Li, Yao, Jing, Jia, Teng and He. This is an open-access article distributed under the terms of the Creative Commons Attribution License (CC BY). The use, distribution or reproduction in other forums is permitted, provided the original author(s) and the copyright owner(s) are credited and that the original publication in this journal is cited, in accordance with accepted academic practice. No use, distribution or reproduction is permitted which does not comply with these terms. 